\title{
MECHANICAL EQUIPMENT SAFETY ASSESSMENT AND FAULT DIAGNOSIS SYSTEM UNDER BIG DATA MINING TECHNOLOGY
}

\author{
Da Wang ${ }^{1}$, Kun Zhang ${ }^{1}$ \\ ${ }^{1}$ School of Computer and Information Technology, Nanyang Normal University, Nanyang 473061, China. \\ Email: zhangkundr473@yeah.net
}

\begin{abstract}
To solve the problem that mechanical equipment fault monitoring is difficult because of the vibration of signals and improve the accuracy of fault location during the operation of mechanical equipment, taking big data mining technology as the core, a set of mechanical equipment safety assessment and fault diagnosis system is designed. Firstly, the design scheme of the system and relevant algorithm improvement of big data mining technology are introduced. Then, on the basis of big data technology, the hardware and software of mechanical equipment fault diagnosis system are designed. Finally, according to the simulation experiment, the performance evaluation of the data optimization algorithm and the analysis of the system simulation obstacle experiment results are carried out. The results show that the Correlation Matching Extension (CME) method and the adaptive set empirical mode decomposition algorithm proposed in this research can reduce the decomposition error of signals, improve the analysis effect of rotating mechanical equipment, and locate the fault characteristics of mechanical equipment quickly. This research can promote the application of big data mining technology in mechanical field.
\end{abstract}

Keywords: Big Data Mining Technology; Mechanical Equipment; Safety Assessment; Fault Diagnosis; CME Method.

\section{Introduction}

With the development of China's economy, the degree of automation of mechanical equipment is getting higher and higher. The improvement of automation of modern mechanical equipment also reflects the importance of safe and stable operation of equipment. In the operating state of mechanical equipment, even a small fault may lead to safety accidents, which may cause large economic losses and relatively serious consequences, such as casualties [1,2].

Therefore, it is urgent to study the safety evaluation and fault diagnosis system of mechanical equipment.

Rotating machinery refers to the mechanical parts that work through the rotating motion of the rotor in a mechanical system, which is a common system in industrial machinery system. Most of the rotating machinery are power machinery.

The position of rotating machinery in mechanical equipment should not be underestimated, which can not only conduct real-time monitoring of mechanical equipment in operation to ensure the safe operation of mechanical equipment, but also timely find and troubleshoot faults, which is a key component to provide important guarantees for enterprise equipment or military equipment [3-5].

At present, for rotating machinery, fault diagnosis has three important tasks: I. fault diagnosis; II. fault location and fault type diagnosis; III. the quantitative diagnosis of fault, mainly unbalanced fault. For the current traditional fault diagnosis technology, it is difficult to complete the above three main tasks, not to mention the diagnosis of key parts of complex mechanical equipment, and no satisfactory solution has been found at present [6].

Currently, the commonly used fault diagnosis methods include signal processing, model analysis, and knowledge discovery, etc. However, these diagnosis methods all have certain limitations and shortcomings [7].

With the development of industrial revolution, industrial intelligence is the goal to be achieved in industry 4.0, that is, to use information technology to promote industrial reform and realize the goal of industrial intelligence. With the development of information technology, artificial intelligence technology, big data technology, and block chain technology have provided the possibility for the development of industrial intelligence. 
Intelligent industry means that industrial manufacturing can put forward higher requirements for online intelligent diagnosis of industrial equipment faults, which requires no frequent stop monitoring in the whole process of the assembly line to ensure the normal running state of the industry.

At present, the technology of big data mining can integrate the data in the operation of industrial machinery and equipment to dig out more useful information. Therefore, it is of great significance to find a highly accurate data mining algorithm for fault diagnosis of mechanical equipment.

To sum up, in order to improve the accuracy and efficiency of mechanical equipment fault diagnosis system, a set of mechanical equipment safety assessment and fault diagnosis system is designed based on big data mining technology.

Firstly, the design scheme of the system and relevant algorithm improvement of big data mining technology are introduced. Then, on the basis of big data technology, the hardware and software of mechanical equipment fault diagnosis system are designed.

Finally, according to the simulation experiment, the performance evaluation of the data optimization algorithm and the analysis of the system simulation obstacle experiment results are carried out. It is expected that this research can promote the development of industrial intelligence.

\section{Methods \\ 2.1 System scheme design}

In this research, the rotating mechanical equipment of wind turbine is selected for safety assessment and fault diagnosis system of mechanical equipment.

The system should consist of three modules: external sensor, signal collector, and upper computer software. The selection of external sensors should be based on different engineering requirements, and different sensors should be selected to obtain different analogue signals during sensor access. The signal collector has to satisfy three conditions: the first is to meet the maximum synchronous acquisition of 8-channel analogue signal input; second, it is necessary to control and set the parameters of each channel signal; the third is to accept the signal data sent by the upper computer and set the parameters of the acquisition channel. The upper computer software part is to display 8channel data, storage, and processing functions.

According to the conception of safety assessment and fault diagnosis system for mechanical equipment, the functions to be realized are summarized.

Table 1 is the functions to be realized for the mechanical equipment safety assessment and fault diagnosis system designed in this research.

Table 1. The function of safety assessment and fault diagnosis system of mechanical equipment designed in this

\begin{tabular}{|l|l|}
\hline Three modules & Functions \\
\hline $\begin{array}{l}\text { External sensor } \\
\text { module }\end{array}$ & $\begin{array}{l}\text { With different types of sensor access capabilities and a wide range of analogue } \\
\text { signal input capabilities }\end{array}$ \\
\hline \multirow{3}{*}{$\begin{array}{l}\text { Signal acquisition } \\
\text { module }\end{array}$} & Capable of data storage, data analysis, and fault diagnosis \\
\cline { 2 - 2 } & $\begin{array}{l}\text { With the ability of signal pre-processing and parallel acquisition of 8-channel } \\
\text { analogue signals }\end{array}$ \\
\cline { 2 - 2 } & Necessary to have high A/D conversion rate and accuracy \\
\hline $\begin{array}{l}\text { Upper computer } \\
\text { software module }\end{array}$ & $\begin{array}{l}\text { Necessary to have advantages of high integration and good expansibility, so as } \\
\text { to improve the optimization }\end{array}$ \\
\hline
\end{tabular}

Figure 1 is the mechanical equipment safety assessment and fault diagnosis system designed in this research. The signal acquisition part between the external sensor part and the upper computer plays a bridge connection part. From the perspective of functional structure, the information acquisition part can be divided into three parts: power supply part, signal acquisition part, and data processing part. The power module provides analogue or digital voltages of different sizes for the entire circuit board. In order to meet the requirements of different sensors, the signal acquisition module needs to set an interface for each sensor. The interface mainly realizes four functions, which are gain to the signal input by the sensor, processing related conditioning circuit and filter, and A/D conversion circuit for the signal after the modulation. Then, the data processing control module (ARM and FPGA) is used to ensure the normal communication with the upper computer. The communication of the upper computer is mainly based on FPGA receiving the control information sent by ARM to configure its register to achieve the purpose of configuring acquisition parameters. Finally, the obtained A/D conversion data is sent to ARM and ARM. Various configured acquisition parameters and other information are received from the upper computer and sent to FPGA. Meanwhile, data obtained from FPGA are sent to the upper computer through network ports and other interfaces.

When selecting the main components of the collector, FET high-resistance operational amplifier 
OPA2134 should be selected as the amplifier for signal acquisition. The amplifier has high input impedance, high co-simulation capability, and highpressure swing rate $(20 \mathrm{~V} / \mathrm{cm})$. The power supply range is $2.5 \sim 18 \mathrm{~V}$, and the adaptable working environment temperature is $-45^{\circ} \mathrm{C} \sim+85^{\circ} \mathrm{C}$, which can meet the requirements of this study.

For ADC, the ADC selected in this research needs to improve the accuracy of the acquisition signal, so ADC with higher resolution is required.

Therefore, AKM company's A/D chip AK5385B is selected in the research. For the FPGA in the collector, XILINX company's xc3s500e-4pq2081 is selected, and for ARM, STM32F407 with cortex-m4 kernel is chosen.
Sensors are very important components of mechanical equipment. As for the acceleration sensor, ICP sensor will be selected in this research. The output voltage signal is in direct proportion to the acceleration, and is completely closed structure, which can effectively reduce external interference. In addition, its characteristics of easy installation and high precision of signal collection also make it widely used.

In the selection of audio sensors, two parameters should be taken into account, namely, sound pressure to represent the amplitude of the sound and tone to represent the frequency of the sound. In combination with the situation in this research, MA231 acoustic signal sensor will be used, and its main technical indicators are shown in Table 2.

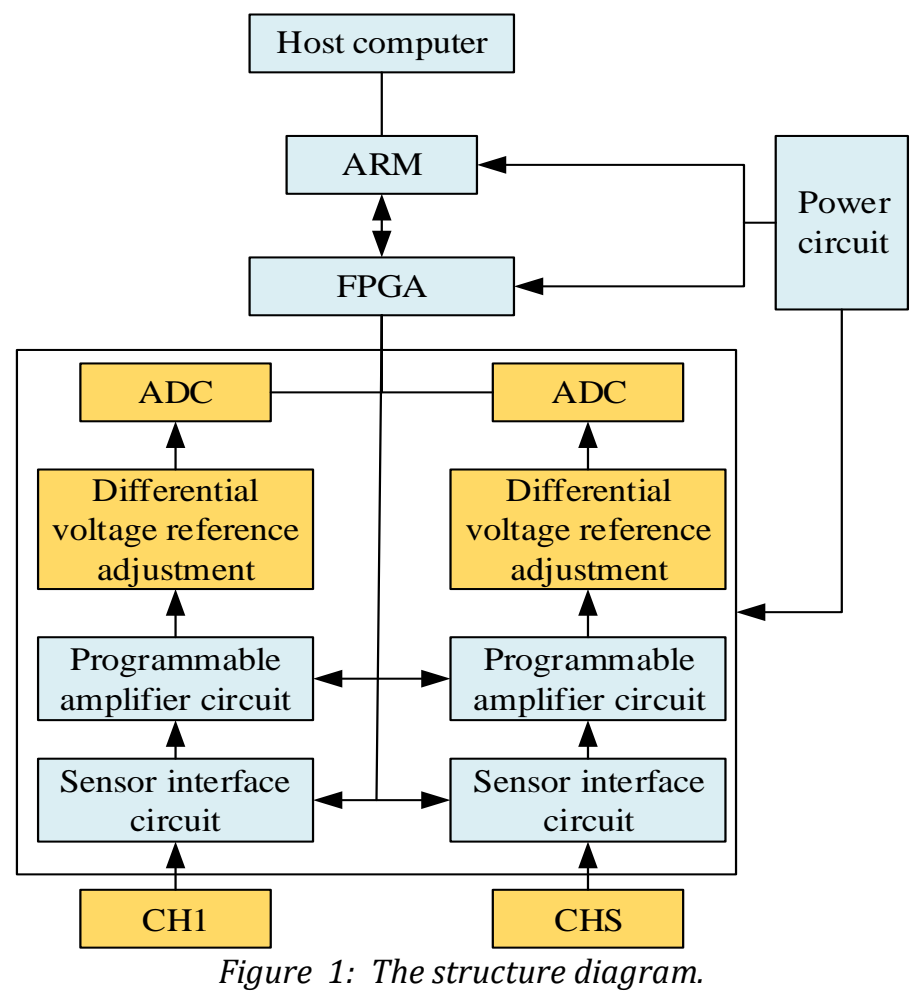

Table 2. Technical indicators summarization of MA231 acoustic signal sensors.

\begin{tabular}{|l|l|l|l|}
\hline Indexes & Value range & Indexes & Value range \\
\hline $\begin{array}{l}\text { Attenuation } \\
(10 \sim 100 \mathrm{~Hz})\end{array}$ & $<0.15 \mathrm{~dB}$ & $\begin{array}{l}\text { Maximum output } \\
\text { voltage }\end{array}$ & ICP $(+1 \sim+250 \mathrm{~mA})$ \\
\hline Input impedance & $20\|0.1(G \Omega)\| \mathrm{pF}$ & Sensitivity & $50 \mathrm{mV} / \mathrm{Pa}$ \\
\hline Output impedance & $<60 \Omega$ & Background noise & $20 \mathrm{~Hz} \sim 2000 \mathrm{~Hz}$ \\
\hline $\begin{array}{l}\text { Frequency } \\
\text { response }\end{array}$ & $20 \mathrm{~Hz} \sim 150000 \mathrm{kHz}$ & $\begin{array}{l}\text { Maximum output } \\
\text { voltage }\end{array}$ & $5 \mathrm{~V}$ \\
\hline
\end{tabular}

\subsection{Related algorithm improvement of big data mining technology}

In this research, Empirical Mode Decomposition (EMD) method is adopted to screen the data of the signal acquisition system. The steps of EMD method are as follows.
The first thing is to make sure that all of the local extrema points that exist on the original signal $x(t)$. Cubic spline interpolation is used to fit the data of extreme point and minimum point.

The maximum point is expressed as $f_{\max }(t)$, the minimum point as $f_{\min }(t)$. The mean of the 
maximum and minimum values is denoted as $m(t)$. And the difference between $x(t)$ and $m(t)$ is denoted as $h_{1}(t)$, which is expressed as Eq. 1.

$$
h_{1}(t)=x(t)-m(t)
$$

Because the real signals are very complex, there are asymmetric waves in $h_{1}(t)$, and there are common fitting defects and new inflection points in data fitting. $h_{1}(t)$ is viewed as new $x(t)$, then Eq. 2 is obtained.

$$
h_{11}(t)-m_{11}(t)=h_{11}(t)
$$

Since the boundary problem has a great influence on the accuracy of EMD decomposition in the process of signal processing, after the EMD method was proposed, the research on boundary suppression has been concerned by many scholars [8].

Relevant scholars put forward the method of processing this case with two sections of data. The main idea of this method is to use algorithms to extend a piece of data outward at both ends of the signal; then the existing sequence is "guarded" in the new sequence, that is, the original endpoint is moved to the new boundary.

This method is used to erase the effect of boundary effect on the original data [9].

At present, there are following types of boundary continuation methods $[10,11]$.

I. Extremum continuation method.

The method should first use the extremum extension method to determine the extremum distribution at both ends of the original sequence. Some algorithms are then used to select the specific extremum to be contiguous at both ends of the original sequence. This method includes direct extremum succession and parallel extremum succession. The direct extremum continuous method gives priority to the accuracy of the fitting curve, which has a lot to do with the extremum point, so it only needs to move the pole at the extremum point, that is, the boundary to a position outside the boundary. The parallel continuation method is mainly used to make the connection lines of the maximum and minimum values near the boundary parallel to each other. According to this law, the position and amplitude of the extended point can be defined subjectively. Due to the existence of a few extreme values at the boundary of the method, the improvement effect for a large range of signals is not obvious [12].

II. Data forecasting.

Combined with the variation characteristics of the whole signal, this method can predict the location and size of the extremum to be extended based on the internal characteristics of the original analogue signal. Because this type of extension integrates the inherent characteristics of the original data, it is a significant improvement over direct extensions and is well applied. However, sometimes the predicted continuous sequence is too short and even the deviation of the continuous sequence is large, which has affected the popularity of this method.

More importantly, prediction usually requires a long learning process [13].

III. Waveform continuation method.

This method is based on the graphic characteristics at the end and beginning of the sequence, and obtains a certain waveform from within the sequence according to relevant laws or features. This method can greatly improve the processing of cyclic sequences, but it is not ideal for processing data with asymmetric boundaries [14].

According to the introduction above, the CME is mainly adopted in the research. This method locates the data segment in a sequence close to the curve at the boundary; then the curve of a certain length is extracted outside the data segment and added to the boundary to obtain the curve of edge extension and make the widened curve consistent with the trend of the original sequence.

The specific steps are as follows:

Step 1: in the sequence of time domain curves, supposing that the left boundary point of curve $x(t)$ is $K_{0}$, a horizontal line is made from the point $K_{1}, K_{2}, K_{3}, \cdots, K_{n}$ at which the curve intersects.

Step 2: with $K_{0}$ as the endpoint, the data segment from right to left through zero and the extreme point is $l o$, and the data segment is calculated.

Step 3: the second step is repeated, taking $K_{1}, K_{2}, K_{3}, \cdots, K_{n}$ as the endpoint, the data segment is intercepted and each data segment is calculated.

Step 4: Eq. 3 is used to find the correlation coefficient $\mathrm{R}_{\mathrm{i}}$ between $l_{0}$ and each data segment $L_{1}, L_{2}, L_{3}, \cdots, L_{n}$. Eq. 3 is expressed as follows.

$$
R_{i}=\frac{\left\langle L_{0}, L_{i}\right\rangle}{\left\|L_{0}\right\|\left\|L_{i}\right\|}
$$

Step 5: finally, the maximum value of the correlation coefficient is found. The corresponding $L_{i}$ is the starting point of the data. Segment $L_{a}$ of the curve containing multiple extrema points to the left of the starting point represents the data continuation of the left endpoint, and the length of segment $L_{a}$ of the curve is set to $l_{a}$.

\subsection{Hardware and software design of mechanical equipment fault diagnosis system}

The overall framework diagram of hardware circuit of mechanical equipment fault safety 
assessment and fault diagnosis system is shown in Figure 2.

It consists of three parts: signal acquisition module, data processing module, and power module.
The design of signal analogue acquisition module has the same function as the system design in this research, which is not described any more.

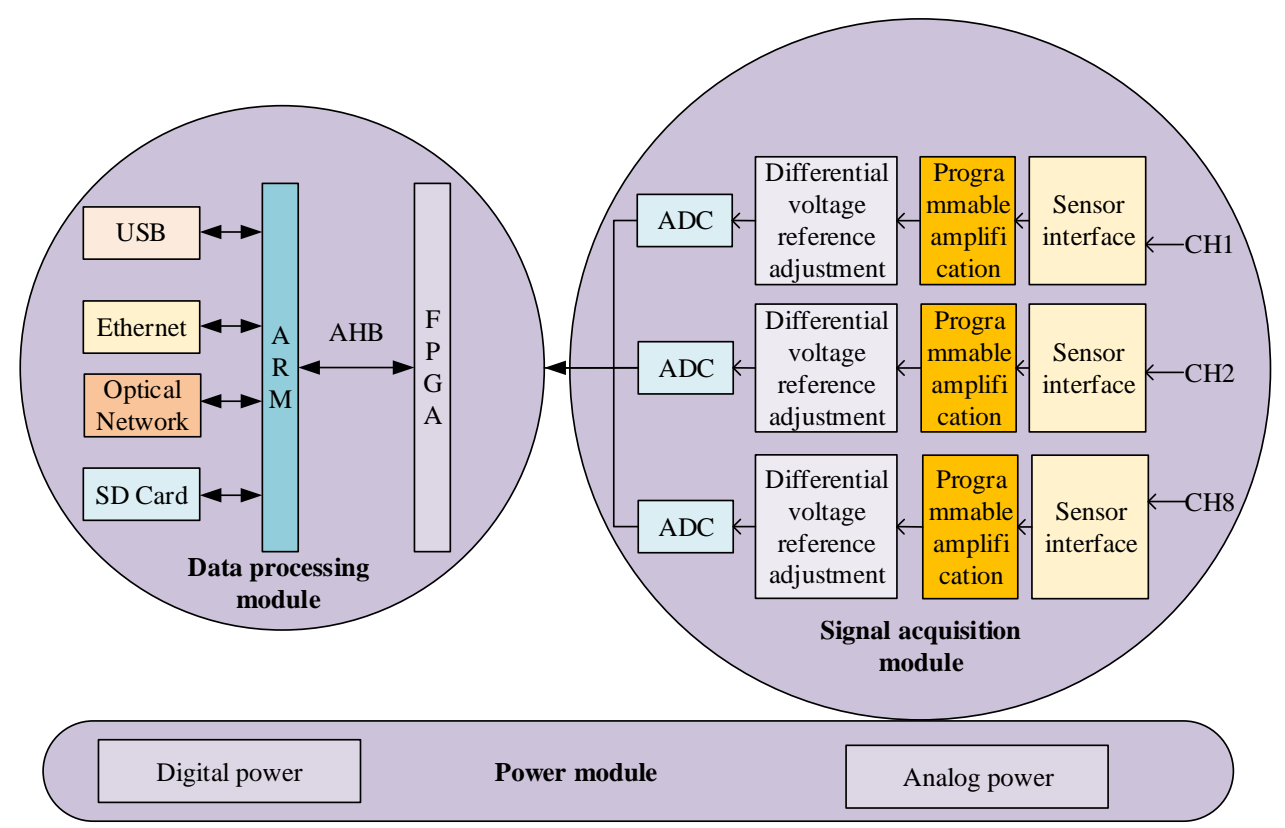

Figure 2: Hardware circuit diagram of mechanical equipment fault safety assessment and fault diagnosis system.

\section{Results and Discussion}

\subsection{Performance evaluation of data mining optimization algorithm}

Firstly, the improved EEMD algorithm is simulated to decompose the original signal. In this research, the original signal is firstly decomposed into the first IMF component through EEMD, and the amplitude coefficient is 0.705 . It is found that the coefficient ratio of Gauss to full-frequency noise is 0.176 , while the EMD is set to 330 .
Figure 3 shows the decomposition results of the improved EEMD method. The better decomposition of the first-order component is the decomposition of the initial signal at $180 \mathrm{~Hz}$, while the second-order component partially reduces the modulation wave.

Compared with the initial signal, the pseudocomponent has a smaller energy level, and the improved EEMD algorithm can effectively decompose the components of the original signal.

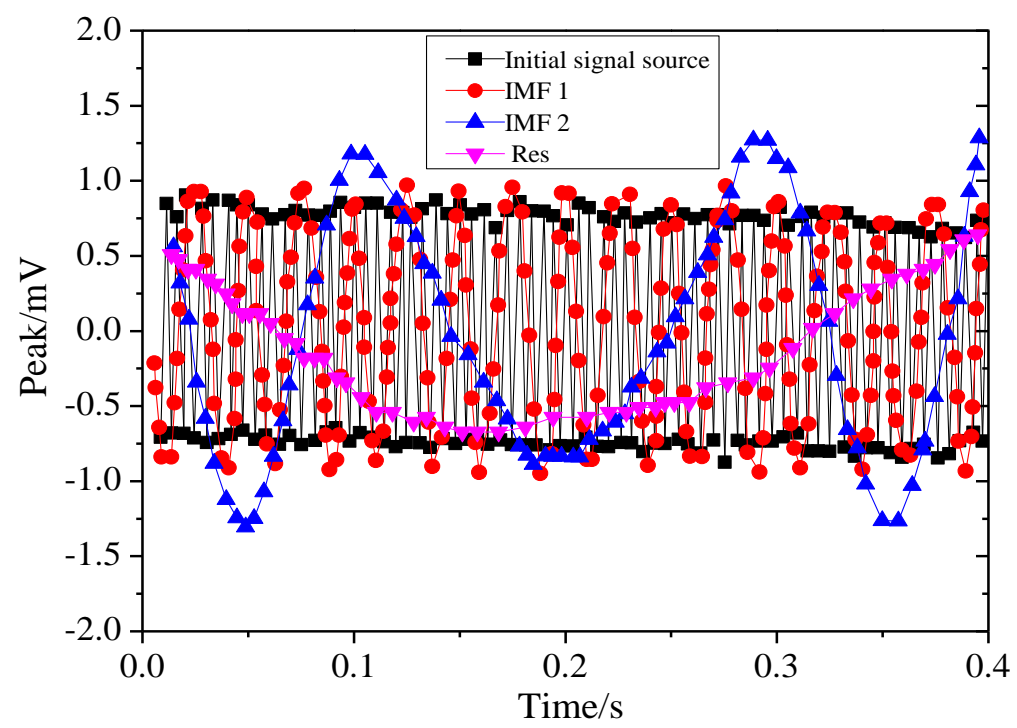

Figure 3: Decomposition results of the improved EEMD method. 
After decomposing the signal, Hilbert transformation is performed on the initial signal.

Figure 4 shows the Hilbert time-frequency diagram of EEMD decomposition of the improved simulation signal.

Figure 5 shows the Hilbert spectrum of EEMD decomposition of the simulation signal. When the unimproved EEMD performs Hilbert spectral decomposition on the sample points, the signals at the end of the EEMD diverge seriously. However, when the improved EEMD performs Hilbert spectral decomposition on the collected signal, the terminal dispersion phenomenon hardly exists. Moreover, the improved EEMD spectrum can clearly distinguish the spectral lines.

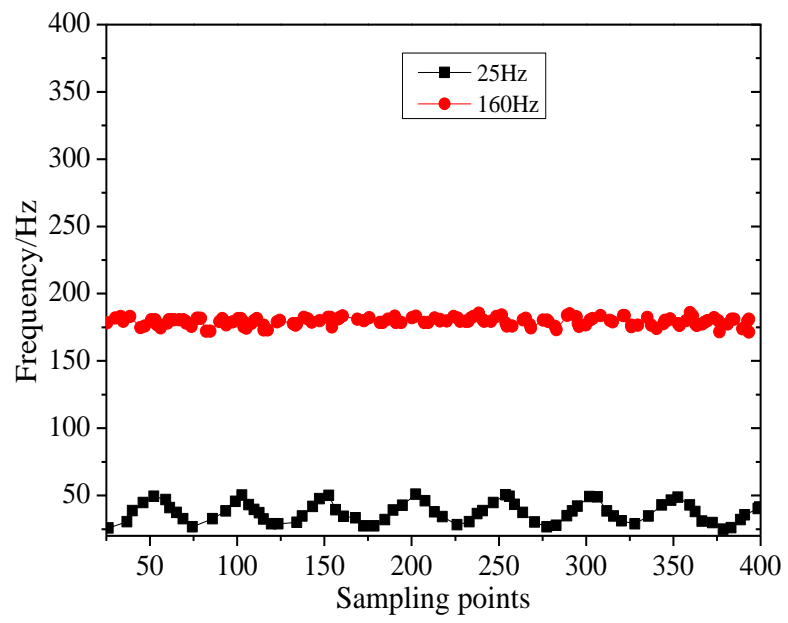

Figure 4: The Hilbert-time spectrum of EEMD decomposition improved by simulation signals.

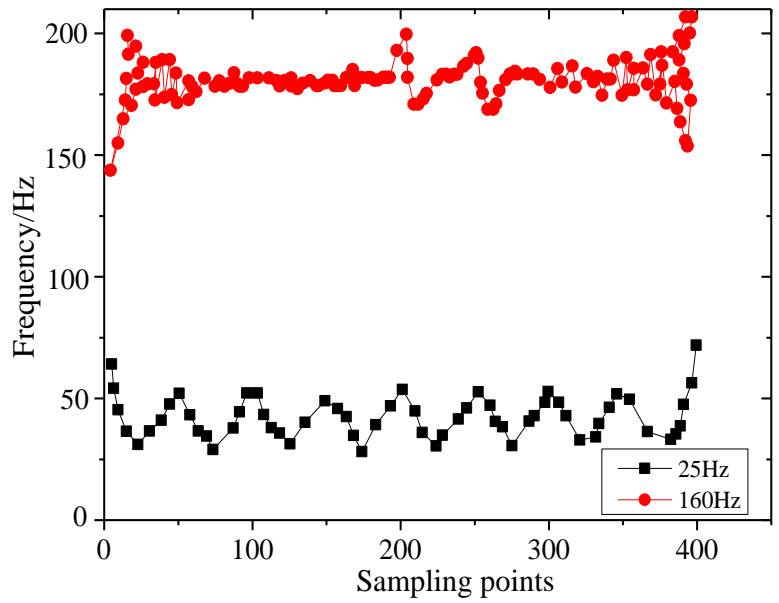

Figure 5: Hilbert - time spectrum decomposed by simulation signal EEMD.

Therefore, the improved algorithm can effectively improve the phenomenon of modal aliasing in the Hilbert time-frequency decomposition of the collected signal, which is superior to the unimproved EEMD algorithm.

\subsection{Analysis of system simulation fault test results}

In order to evaluate the safety of the designed mechanical equipment and verify the effectiveness of the fault diagnosis system, the fault of the rotor mechanical equipment is simulated. In order to realize the unbalance of rotor mechanical equipment, the artificial method is adopted. A weight is added to one side of the rotor disk, resulting in an imbalance between the rotor's left and right sides. Then the rotor speed is kept at $1025 \mathrm{r} / \mathrm{min}$, the sampling signal frequency is $1 \mathrm{kHz}$, and the rotation frequency is $17.1 \mathrm{~Hz}$. The collected vibration signal data are shown in Figure 6.

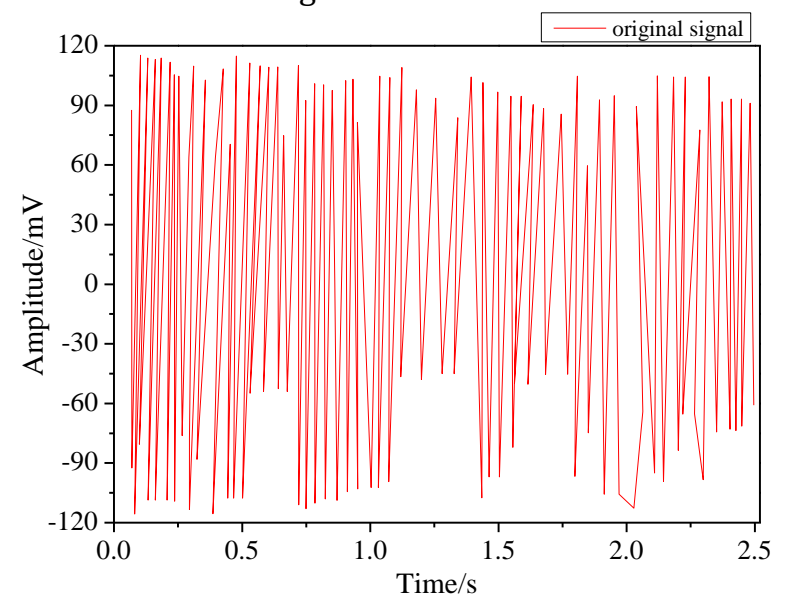

Figure 6: The measured vibration signal.

Under normal circumstances, the signal waves collected by this system are very messy.

There are two main reasons for this phenomenon. On the one hand, it is possible that the rotor mechanical equipment is malfunctioning during operation.

The other reason is that the signal collects a lot of noise in the process of acquisition and reading. Based on the above two reasons, the morphology of the original waveform can no longer be seen from the measured signal spectrum. Therefore, in this research, the frequency spectrum is transformed, and the main signal is concentrated at $17.1 \mathrm{~Hz}$.

In order to further analyse the components inside the signal, the CEM is adopted to expand the length of the collected signal. Then the signal is decomposed by EEMD method.

Figure 7 shows the Hilbert - time spectrum of EEMD decomposition based on CME. Figure 8 shows the marginal spectrum of the improved EEMD decomposition based on CME. As shown in Figure 7, the Hilbert frequency spectrum of EEMD decomposition based on CME proposed in this research can clearly reflect each frequency component in the signal process. Especially in the fundamental frequency component, the frequency modulation component can be highlighted. 


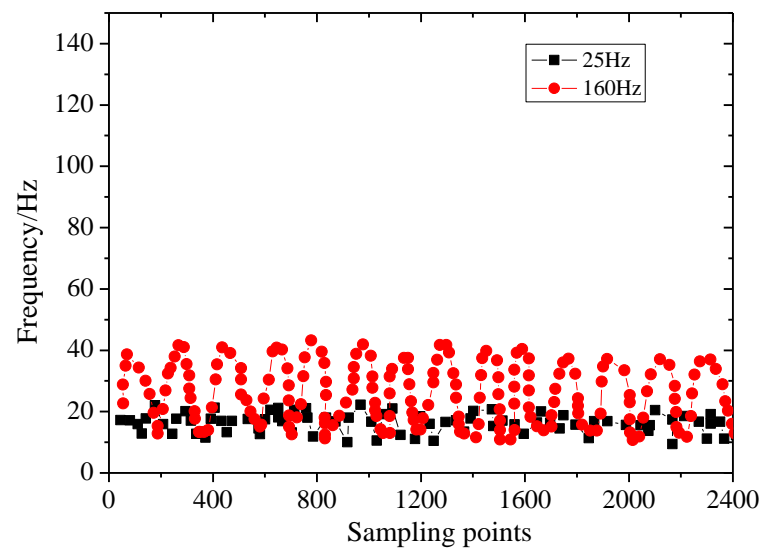

Figure 7: EEMD decomposition results based on CME improvement.

Moreover, the spectral lines are clear, which effectively reduces the generation of pseudocomponents and weakens the problem of mode aliasing. Moreover, it can be seen from the map that the fluctuation of both ends of the signal is relatively weak, and there is no interleaving and overlapping among components, which has a good inhibitory effect on the fluctuation of the signal boundary.

Figure 8 is the marginal spectrum of the original signal. It can be seen from the signal spectrum that the amplitude appears near the fundamental frequency of the rotor mechanical equipment, and the energy displayed by the whole signal wave is mainly in the fundamental frequency. Therefore, the fault characteristics caused by the unbalance of the rotor in the process of rotation are accurately located.

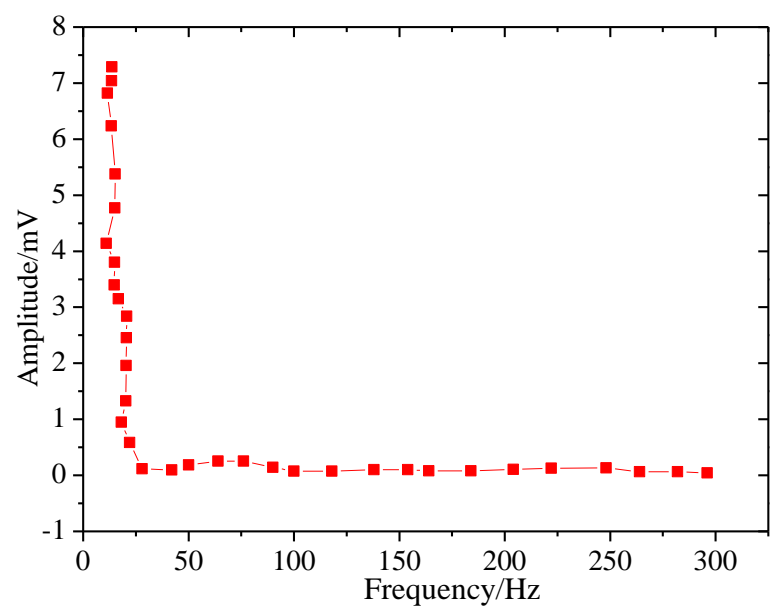

Figure 8: The marginal spectrum of EEMD decomposition based on CME improvement.

In the absence of faults, the spectrum diagram of the vibration signal shows that the amplitude of the vibration signal should be 1 octave at most, then 2 octaves.

Therefore, the method proposed in this research can accurately locate the fault.

\section{Conclusions}

In order to ensure the safe operation of mechanical equipment in different fields and improve the accuracy of mechanical fault monitoring, the big data mining technology is used to design a set of mechanical equipment safety assessment and fault diagnosis system. First of all, the design scheme of mechanical equipment safety assessment and fault diagnosis system is introduced, and the selection of various components in the scheme is explained in detail; then, relevant algorithms of big data mining technology are improved and hardware and software of mechanical equipment fault diagnosis system are designed; finally, the simulation experiment is used to evaluate the performance of the data mining algorithm used in the mechanical equipment safety assessment and fault diagnosis system. Experiments show that the improved EEMD decomposition algorithm proposed in this research can effectively improve the phenomenon of modal aliasing in the Hilbert time-frequency decomposition of the collected signal, which is superior to the unimproved EEMD algorithm. According to the analysis of system simulation fault test results, the improved EEMD decomposition algorithm based on CME proposed in this research can effectively locate fault characteristics.

The research provides a good idea for the evaluation and diagnosis of mechanical equipment failure, but the research still has certain limitations. This research studies the mechanism of the HilbertHuang transformation method, although the mechanism of the Hilbert-Huang transformation method shows a good advantage in nonlinear signal processing, its theory is not yet perfect. Therefore, further optimization is still needed for EMD decomposition cut-off conditions and anti-modal aliasing methods.

\section{References}

[1] H. Daki, H. A. El, A. Aqqal, et al. "Big Data management in smart grid: concepts, requirements and implementation." Journal of Big Data, vol.4, no.1, pp. 1-19, 2017.

[2] Y. Xu, Y. Sun, J. Wan, et al. "Industrial big data for fault diagnosis: Taxonomy, review, and applications." IEEE Access, vol.5, pp.17368 17380, 2017.

[3] D. Zhang. "High-speed train control system big data analysis based on the fuzzy rdf model and uncertain reasoning." International Journal of Computers Communications \& Control, vol.12, no.4, pp. 577-591, 2017.

[4] Y. Lu. "Industry 4.0: A survey on technologies, applications and open research issues." Journal of Industrial Information Integration, vol.6, pp.1-10, 2017. 
[5] M. Marjani, F. Nasaruddin, A. Gani, et al. "Big IoT data analytics: architecture, opportunities, and open research challenges." IEEE Access, vol.5, pp. 5247-5261, 2017.

[6] C. V. Asche, B. Seal, K. H. Kahler, et al. "Evaluation of healthcare interventions and big data: review of associated data issues." Pharmacoeconomics, vol.35, no.8, pp.759-765, 2017.

[7] Y. Zhang, S. Ren, Y. Liu, et al. "A big data analytics architecture for cleaner manufacturing and maintenance processes of complex products." Journal of Cleaner Production, vol.142, pp. 626-641, 2017.

[8] M. Du, K. Wang, Y. Chen, et al. "Big data privacy preserving in multi-access edge computing for heterogeneous Internet of Things." IEEE Communications Magazine, vol.56, no.8, pp. 6267, 2018.

[9] C. F. Chien, C. W. Liu, S. C. Chuang. " Analysing semiconductor manufacturing big data for root cause detection of excursion for yield enhancement." International Journal of Production Research, vol.55, no.17, pp. 5095 5107, 2017.
[10] M. G.Kibria, K. Nguyen, G. P. Villardi, et al. "Big data analytics, machine learning, and artificial intelligence in next-generation wireless networks." IEEE access, vol.6, pp.32328-32338, 2018.

[11] C. Li, S. Lin, F. Xu , et al. "Short-term wind power prediction based on data mining technology and improved support vector machine method: A case study in Northwest China." Journal of cleaner production, vol. 205, pp. 909-922, 2018.

[12] F. Gu, B. Ma, J. Guo, et al. "Internet of things and Big Data as potential solutions to the problems in waste electrical and electronic equipment management: An exploratory study." Waste Management, vol.68, pp. 434-448, 2017.

[13] L. R. Nair, S. D. Shetty. "Applying spark based machine learning model on streaming big data for health status prediction." Computers \& Electrical Engineering, vol.65, pp. 393-399, 2018.

[14] M. Mohammadi, A. Al-Fuqaha. "Enabling cognitive smart cities using big data and machine learning: Approaches and challenges." IEEE Communications Magazine, vol.56, no.2, pp. 94-101, 2018. 